\title{
Successful intracytoplasmic sperm injection with testicular spermatozoa using pentoxifylline and HOS from a male with necrozoospermia: a case report
}

\author{
Usharani Nampalli ${ }^{1}$, Syed Fyzullah ${ }^{1}$, Chinmayie R. $^{2}$, Asha Srinivasan Vijay ${ }^{2 *}$, Rajsri T. R. ${ }^{3}$
}

\author{
${ }^{1}$ Department of Embryology, Garbhagudi IVF Centre, Bangalore, Karnataka, India \\ ${ }^{2}$ Department of Reproductive Medicine, Garbhagudi IVF Centre Bangalore, Karnataka, India \\ ${ }^{3}$ Department of Community Medicine, Evidencian Research Association, Karnataka, India
}

Received: 04 February 2021

Revised: 27 March 2021

Accepted: 31 March 2021

\section{*Correspondence:}

Dr. Asha Srinivasan Vijay,

E-mail: ashasvijay@gmail.com

Copyright: (c) the author(s), publisher and licensee Medip Academy. This is an open-access article distributed under the terms of the Creative Commons Attribution Non-Commercial License, which permits unrestricted non-commercial use, distribution, and reproduction in any medium, provided the original work is properly cited.

\begin{abstract}
The study was done to compare fertilization, cleavage, and pregnancy outcome of a man affected with necro zoospermia using pentoxifylline and a hypoosmotic swelling (HOS) test to select viable spermatozoa from testicular biopsy specimens. A patient was diagnosed with necrozoospermia by semen analysis, and testicular spermatozoa were injected intracytoplasmically, using pentoxifylline and HOS (Hypo osmotic swelling test) and the following ICSI results were observed. Significantly higher fertilization rates (pentoxifylline $100 \%$ vs HOS $80 \%$ ) were observed. There was no significant difference observed in cleavage rates among both groups. Finally, a healthy female infant with birth weight $3 \mathrm{~kg}$ was delivered at the second frozen embryo transfer cycle.it was found that selecting viable spermatozoa using pentoxifylline was more effective in fertilization and pregnancies than obtaining it through a HOS test. ICSI is effective for necrozoospermic man.
\end{abstract}

Keywords: Necrozoospermia, Intracytoplasmic sperm injection, Testicular sperm, HOS, Pentoxyfylline

\section{INTRODUCTION}

Necrospermia (or necrozoospermia) is a condition characterized by low percentage of live sperms and a high percentage of immotile spermatozoa in semen. ${ }^{1}$ The only way to diagnosis the Necrozoospermia is with a semen analysis. During ICSI necrozoospermic sperms can't be injected. The best available management for necrozoospermia is testicular sperm extraction (TESE) with ICSI using pentoxifylline or HOS. ${ }^{2}$

\section{CASE REPORT}

A 32-year-old female presented with primary infertility for nine years and was seeking treatment at Garbhagudi IVF centre. His Semen analysis showed necrozoospermia. On evaluation, he had no history of respiratory symptoms. No apparent organic anomalies were found during a physical examination, and ultrasound examination revealed normal male reproductive organs. The Biochemical profile of the patient was as follows serum follicle-stimulating hormone (FSH $1.94 \mathrm{mIU} / \mathrm{ml}$ ), luteinizing hormone (LH 6.42 $\mathrm{mIU} / \mathrm{ml}$ ), and testosterone (T $390 \mathrm{ng} / \mathrm{ml}$ ) were within normal ranges, and his $\mathrm{Y}$-chromosome microdeletion report was normal.

Following is the semen analysis report: the evaluation of the semen samples were done based on WHO guidelines, several semen analyses showed sperm concentrations of 10-14 million $\mathrm{ml}^{-1}$, the volume of 1.0-2.4 ml, with seminal $\mathrm{pH}$ at 7.4. All semen analysis had complete immotile sperms, and the majority was abnormal with normal morphology of $1 \%$. Eosin nigrosin staining method was performed to assess the vitality of the sperm, and only $12 \%$ 
of the sperm found to have an intact structural membrane (WHO reference limit- 58\%) (Figure 1). Couple were counselled for ICSI procedure in view of necrozoospermia.

\section{Stimulation and ICSI procedures}

After down-regulation with OCP in the previous cycle, on day 2 of the cycle, transvaginal ultrasound was done, and AFC was found to be 14, and stimulation was started. On stimulation day four antagonist started as per flexible protocol and continued till day 11 of the cycle, where 11 follicles were more than $17 \mathrm{~mm}$, and the trigger was given using injection lupride $2 \mathrm{mg}$ and $1 \mathrm{mg}$ administered 12 hours apart. Ovum pick up performed $34.5 \mathrm{hrs}$ after the trigger and retrieved 13 oocytes, of which 10 were mature (MII). In view of high E2 and P4 levels on the day of trigger freeze, all strategy was performed. Transcervical frozen embryo transfer was performed 3months after the oocytes were retrieved and planned for blastocyst transfer and the same have been transferred. Pregnancy was confirmed by two HCG tests conducted 14 days after embryo transfer, and clinical pregnancy was determined by a fetal heartbeat on ultrasound screening after 35 days.

On the Oocytes retrieval day, no motile sperm was found in the ejaculate. Testicular spermatozoa were obtained by needle aspiration, which also showed $100 \%$ immotile sperms. Hence, HOS was done for $100 \mu$ of the sample and could get few viable sperms. Since only a few sperms were found to be viable on HOS, pentoxifylline was used to the rest of the TESE suspension (Figure 2).

The use of pentoxifylline (PTX) allows a more reliable selection of live and viable spermatozoa. Fertilization, cleavage, and clinical pregnancy rates were almost similar to those with the ejaculated motile sperm. To facilitate sperm-activation, we added 1.5-2.0 $\mu \mathrm{l}$ PTX to the spermcontaining drop (approx. 30-40 $\mu \mathrm{l}$ /dilution 1:20) in sperm preparation-medium inside the Petri-dishes. PTX was added to the opposing side from where the sperms are to be aspirated and waited for 10 minutes. The activating effects of PTX initiates after a few minutes and lasts for up to one hour.
Half of the oocytes were injected with sperm treated with HOS and another half with Pentoxifylline. The Oocytes injected with Pentoxifylline achieved $100 \%$ (5/5) fertilization rate, and with HOS achieved $80 \%$ (4/5). frozen embryo transfer with pentoxifylline treated embryos) was planned. A fetal heartbeat was detected by ultrasound at the 32 nd day after transfer. A healthy, 3300 $\mathrm{g}$ female baby was delivered at 40 weeks of gestation. The ICSI results are summarized in Table 1.

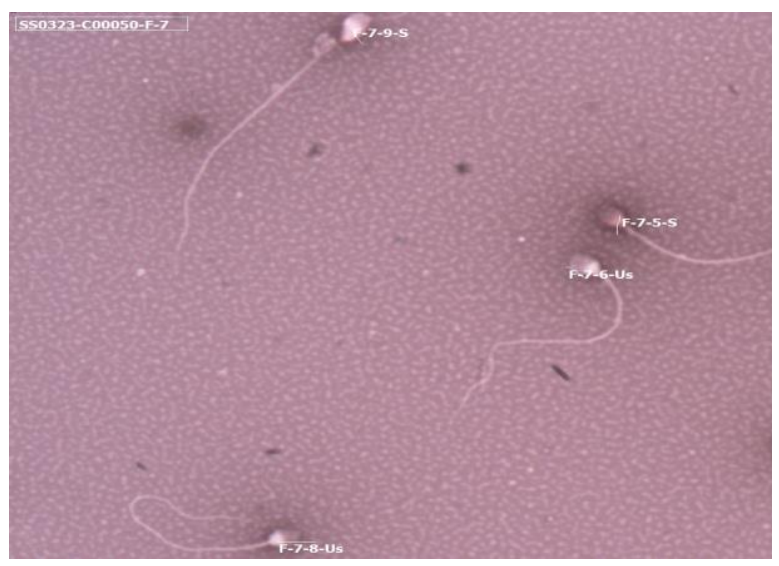

Figure 1: Appearance on viability assessment slide.

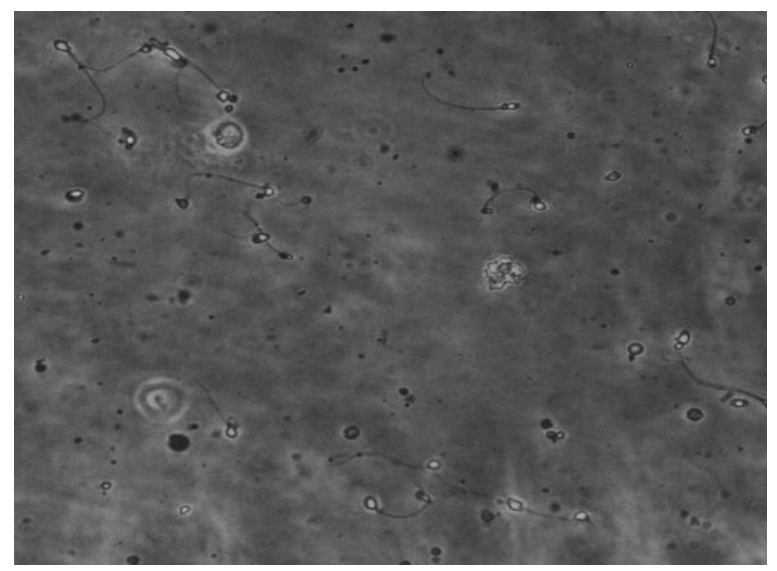

Figure 2: Hypoosmotic swelling test.

Table 1: Intracytoplasmic sperm injection outcomes of the couple.

\begin{tabular}{|llllllll|}
\hline $\begin{array}{l}\text { Intervention } \\
\text { PTX }\end{array}$ & $\begin{array}{l}\text { Number of oocytes } \\
\text { injected }\end{array}$ & $\begin{array}{l}\text { Number of } \\
\text { oocytes fertilized }\end{array}$ & $\begin{array}{l}\text { Number of } \\
\text { embryos cleaved }\end{array}$ & $\begin{array}{l}\text { Quality of the embryos on } \\
\text { day 3 }\end{array}$ & GI & GII & GIII \\
\hline HOS & 5 & 5 & 5 & 4 & 1 & 0 \\
\hline
\end{tabular}

The embryo grades are cell number, fragmentation, and symmetry, respectively, according to the criterion by Isthanbul consensus.

\section{DISCUSSION}

Necrospermia is defined by a percentage of living spermatozoa inferior to $58 \%$ and can be related to male infertility. ICSI provides an effective therapeutic method for patients with necrozoospermia, but selecting live sperm among immotile sperm is crucial for successful ICSI. A randomly selected immotile ejaculated spermatozoa would negatively influence the reproductive results. When no motile sperm is present, the hypoosmotic swelling (HOS) test is valuable to identify live sperm. However, HOS has 
shown high false positive rates. ${ }^{3}$ Adding Pentoxyfylline to the Testicular sperm was effective for ICSI in case of $100 \%$ immotility along with $\mathrm{HOS}^{4}$ Pentoxyfylline is a non-specific phosphodiesterase (PDE) inhibitor of the methylxanthine group. Pentoxyfylline facilitates the motility of the sperm by inhibiting the breakdown of cyclic adenosine monophosphate (cAMP). It is well known that Intracellular cAMP plays a major role in sperm motility. The molecular basis of cAMP regulation is based on protein phosphorylation, especially for capacitation. In addition to the effects on sperm motility, Tesarik et al. reported that PTX augments the acrosome reaction. ${ }^{5}$ It has also been reported that pentoxifylline has no cytotoxic or genotoxic effects. ${ }^{4}$

\section{CONCLUSION}

ICSI with testicular sperm (TESE) using pentoxifylline or HOS is an effective method to achieve a pregnancy in those men affected with necrozoospermia pentoxifylline allows more reliable selection of viable spermatozoa. The use of viable immotile spermatozoa after pentoxifylline exposure during selection process has showed a considerable increase in the fertilization rates and clinical pregnancies. adverse effects such as on embryo cleavage or morphology were not observed. this study concludes that pentoxifylline exposure effectively selects viable sperm out of the nonmotile spermatozoa, and the selected viable sperms can be used to accomplish satisfactory fertilization, cleavage, and pregnancy rates in necrozoospermia.

\section{ACKNOWLEDGMENTS}

We acknowledge the technical support in data entry, analysis and manuscript editing by Evidencian Research Associates.
Funding: No funding sources Conflict of interest: None declared

Ethical approval: Not required

\section{REFERENCES}

1. World Health Organization. WHO laboratory manual for the examination and processing of human semen. 5th ed. 2010.

2. Negri L, Patrizio P, Albani E, Morenghi E, Benaglia $\mathrm{R}$, Desgro $\mathrm{M}$, et al. ICSI outcome is significantly better with testicular spermatozoa in patients with necrozoospermia: a retrospective study. Gynecol Endocrinol. 2014;30(1):48-52.

3. Casper RF, Meriano JS, Jarvi KA, Cowan L, Lucato ML. The hypo-osmotic swelling test for selection of viable sperm for intracytoplasmic sperm injection in men with complete asthenozoospermia. Fertil Steril. 1996;65(5):972-6.

4. Mangoli V, Mangoli R, Dandekar S, Suri K, Desai S. Selection of viable spermatozoa from testicular biopsies: a comparative study between pentoxifylline and hypoosmotic swelling test. Fertil Steril. 2011;95(2):631-4.

5. Tesarik J, Mendoza C, Carreras A. Effects of phosphodiesterase inhibitors caffeine and pentoxifylline on spontaneous and stimulus-induced acrosome reactions in human sperm. Fertil Steril. 1992;58(6):1185-90.

Cite this article as: Nampalli U, Fyzullah $S$, Chinmayie R, Vijay AS, Rajsri TR. Successful intracytoplasmic sperm injection with testicular spermatozoa using pentoxifylline and HOS from a male with necrozoospermia: a case report. Int $\mathrm{J}$ Reprod Contracept Obstet Gynecol 2021;10:2078-80. 